\title{
Acid Sphingomyelinase Gene Deficiency Ameliorates the Hyperhomocysteinemia-Induced Glomerular Injury in Mice
}

\author{
Krishna M. Boini, ${ }^{*}$ Min Xia, ${ }^{*}$ Caixia Li, ${ }^{*}$ \\ Chun Zhang, ${ }^{*}$ Lori P. Payne, ${ }^{*}$ Justine M. Abais, ${ }^{*}$ \\ Justin L. Poklis, ${ }^{*}$ Philip B. Hylemon, ${ }^{\dagger}$ and \\ Pin-Lan Li ${ }^{*}$ \\ From the Departments of Pharmacology and Toxicology," and \\ Microbiology and Immunology, ${ }^{\dagger}$ Medical College of Virginia \\ Campus, Virginia Commonwealth University, Richmond, \\ Virginia
}

Hyperhomocysteinemia (hHcys) enhances ceramide production, leading to the activation of NADPH oxidase and consequent glomerular oxidative stress and sclerosis. The present study was performed to determine whether acid sphingomyelinase (Asm), a ceramide-producing enzyme, is implicated in the development of hHcys-induced glomerular oxidative stress and injury. Uninephrectomized Asmknockout $\left(\mathrm{Asm}^{-/-}\right)$and wild-type $\left(\mathrm{Asm}^{+/+}\right)$mice, with or without Asm short hairpin RNA (shRNA) transfection, were fed a folate-free (FF) diet for 8 weeks, which significantly elevated the plasma Hcys level compared with mice fed normal chow. By using in vivo molecular imaging, we found that transfected shRNAs were expressed in the renal cortex starting on day 3 and continued for 24 days. The FF diet significantly increased renal ceramide production, Asm mRNA and activity, urinary total protein and albumin excretion, glomerular damage index, and NADPH-dependent superoxide production in the renal cortex from $\mathrm{Asm}^{+/+}$mice compared with that from $\mathrm{Asm}^{-1-}$ or Asm shRNA-transfected wild-type mice. Immunofluorescence analysis showed that the FF diet decreased the expression of podocin but increased desmin and ceramide levels in glomeruli from $\mathrm{Asm}^{+/+}$mice but not in those from $\mathrm{Asm}^{-/-}$and Asm shRNA-transfected wild-type mice. In conclusion, our observations reveal that Asm plays a pivotal role in mediating podocyte injury and glomerular sclerosis associated with NADPH oxidase-associated local oxidative stress during hHcys. (Am J Pathol 2011, 179:2210-2219; DOI: 10.1016/j.ajpath.2011.07.019)
Hyperhomocysteinemia (hHcys) is an important pathogenic factor in the progression of end-stage renal disease and in the development of cardiovascular complications related to end-stage renal disease., ${ }^{1,2}$ Hcys induces extracellular matrix accumulation and inhibits its degradation in glomeruli, ultimately leading to glomerulosclerosis and loss of renal function. ${ }^{2-4}$ In addition, our recent studies ${ }^{5}$ revealed that hHcys initiates glomerular damage by inducing podocyte injury. Furthermore, several studies ${ }^{2,6-10}$ have also demonstrated that local oxidative stress mediated by NADPH oxidase (Nox) is importantly involved in the progression of glomerular injury associated with hHcys. However, how the local oxidative stress is activated and thereby results in glomerular injury during hHcys has not yet been fully elucidated.

Previous studies ${ }^{11-19}$ have reported that sphingolipids (mainly ceramide) participate in signal transduction, cell membrane formation, and plasma lipoprotein metabolism, all of which have an impact on the development of atherosclerosis and other sclerotic diseases, such as insulin resistance, obesity, Alzheimer's disease, and cystic fibrosis. Ceramide production is mainly mediated via the hydrolysis of membrane sphingomyelin by various sphingomyelinases, such as acid sphingomyelinase (Asm) or neutral sphingomyelinase, or by de novo synthesis via serine palmitoyltransferase and ceramide synthase. ${ }^{20}$ Ceramide is subsequently metabolized into sphingosine by ceramidases, and sphingosine can be further converted to sphingosine-1-phosphate via sphingosine kinase, ${ }^{20}$ in response to a variety of stimuli, including pro-inflammatory cytokines, oxidative stress, and increased levels of free fatty acids. In addition, ceramide is considered a critical signaling molecule mediating the

Supported by grants from the NIH (DK54927, HL091464, HL57244, and HL075316).

Accepted for publication July 29, 2011

Supplemental material for this article can be found at http://ajp. amjpathol.org or at doi: 10.1016/j.ajpath.2011.07.019.

Address reprint requests to Pin-Lan Li, M.D., Ph.D., Department of Pharmacology and Toxicology, Medical College of Virginia Campus, Virginia Commonwealth University, 410 N. $12^{\text {th }}$ St., Richmond, VA 23298. E-mail: pli@vcu.edu. 
activation of NADPH oxidase in different cells and tissues. ${ }^{4}$ Enhanced plasma Hcys concentrations increase the ceramide production and NADPH activity in the kidney of hyperhomocysteinemic rats. Inhibition of ceramide production improved glomerular injury in those hyperhomocysteinemic rats. ${ }^{4}$ However, it remains unknown whether alterations of Asm gene expression and regulation are implicated in the development of hHcys-induced glomerular oxidative stress and injury, ultimately resulting in glomerulosclerosis.

An inherited deficiency of Asm activity results in the type $A$ and $B$ forms of Niemann-Pick disease, and Asm-knockout mice are resistant to radiation ${ }^{21}$ and other forms of stressinduced apoptosis. ${ }^{22}$ Asm inhibition has rendered cells and animals resistant to the apoptotic effects of diverse stimuli, including Fas-CD95, ${ }^{23}$ ischemia, ${ }^{24}$ radiation, ${ }^{25}$ chemotherapy, ${ }^{26}$ and tumor necrosis factor- $\alpha .{ }^{27}$ Asm knockout or Asm inhibition had protective action during lung inflammation and fibrosis, ${ }^{28}$ cystic fibrosis, ${ }^{29,30}$ obesity and associated glomerular injury, ${ }^{31}$ liver fibrogenesis, ${ }^{32}$ and renal fibrosis. ${ }^{33}$

The present study hypothesized that Asm gene deficiency protects glomeruli from hHcys-induced glomerular oxidative stress and injury and thereby ameliorates glomerulosclerosis under such pathological conditions. To test this hypothesis, we first performed a series of experiments using $\mathrm{Asm}^{-1-}$ and their wild-type (WT) littermates fed the normal chow or folate-free (FF) diet to determine whether lack of the Asm gene alters renal ceramide production, glomerular local oxidative stress, and podocyte injury in mice during hHcys. Then, we locally silenced the renal Asm gene using short hairpin RNA (shRNA) and observed the effects of renal Asm deficiency on hHcys-induced glomerular oxidative stress and corresponding injury. Our results demonstrate that an Asm gene defect or deficiency in the kidney improves the hHcys-induced local oxidative stress and glomerular injury, ultimately preventing glomerulosclerosis.

\section{Materials and Methods}

\section{Animals}

Eight-week-old male C57BL/6J WT Asm ${ }^{-1-}$ mice and their WT littermates were used in the present study. To speed up the damaging effects of hHcys on glomeruli, all mice were uninephrectomized, as previously described. ${ }^{4,5}$ This model has induced glomerular damage unrelated to the uninephrectomy and arterial blood pressure but specific to hHcys. ${ }^{5}$ After a 1-week recovery period from uninephrectomy, mice were fed either a normal chow or an FF diet (Dyets Inc., Bethlehem, PA) for 8 weeks to induce hHcys. In another series of $\mathrm{C} 57 \mathrm{BL} / 6 \mathrm{~J}$ mice, Asm shRNA or a scrambled shRNA (Sigma, St Louis, MO) plasmid with a luciferase expression vector was cotransfected into the kidneys via intrarenal artery injection using the ultrasonographic microbubble system, as previously described. ${ }^{2}$ After delivery of plasmids into the kidney, these uninephrectomized mice were maintained on a normal or an FF diet for 8 weeks. All protocols were approved by the Institutional Animal Care and Use Committee of Virginia Commonwealth University (Richmond, VA).

\section{HPLC Analysis}

Plasma and renal tissue Hcys levels were measured by the high-performance liquid chromatography (HPLC) method, as previously described. ${ }^{5,34}$ A $100-\mu \mathrm{L}$ plasma sample or standard solution mixed with $10 \mu \mathrm{L}$ of internal standard, thioglycolic acid (2.0 mmol/L) solution, was treated with $10 \mu \mathrm{L}$ of $10 \%$ tri-n-butylphosphine solution in dimethylformamide at $4^{\circ} \mathrm{C}$ for 30 minutes. Then, $80 \mu \mathrm{L}$ of ice-cold $10 \%$ trichloroacetic acid in $1 \mathrm{mmol} / \mathrm{L}$ EDTA was added and centrifuged to remove proteins in the sample. The supernatant, $100 \mu \mathrm{L}$, was transferred into the mixture of $20 \mu \mathrm{L}$ of $1.55 \mathrm{~mol} / \mathrm{L}$ sodium hydroxide, $250 \mu \mathrm{L}$ of 0.125 $\mathrm{mol} / \mathrm{L}$ borate buffer $(\mathrm{pH}$ 9.5), and $100 \mu \mathrm{L}$ of $1.0 \mathrm{mg} / \mathrm{mL}$ 4-fluoro-7-aminosulfonylbenzofurazan solution. The resulting mixture was incubated at $60^{\circ} \mathrm{C}$ for 30 minutes to accomplish derivatization of thiols. HPLC was performed with an HP 1100 series instrument (Agilent Technologies, Waldbronn, Germany) equipped with a binary pump, a vacuum degasser, a thermostated column compartment, and an autosampler (Agilent Technologies). Separation was performed at an ambient temperature on an analytical column, Supelco LC-18-DB (Supelco, Bellefonte, PA; $150 \times 4.6 \mathrm{~mm}$ i.d., $5 \mu \mathrm{m}$ particle size) with a Supelcosil LC-18 guard column (Supelco; $20 \times 4.6 \mathrm{~mm}$ i.d., $5 \mu \mathrm{m}$ particle size). Fluorescence intensities were measured with an excitation wavelength of $385 \mathrm{~nm}$ and an emission wavelength of $515 \mathrm{~nm}$ by a Hewlett-Packard model 1046A fluorescence detector (Agilent Technologies). The peak area of the chromatographs was quantified with a Hewlett-Packard 3392 integrator (Agilent Technologies). The analytical column was eluted with $0.1 \mathrm{~mol} / \mathrm{L}$ potassium dihydrogen phosphate buffer $(\mathrm{pH}$ 2.1) containing $6 \%$ acetonitrile $(\mathrm{v} / \mathrm{v})$ as the mobile phase, with a flow rate of $2.0 \mathrm{~mL} /$ minute.

\section{Morphological Examination Findings}

The fixed kidneys were paraffin embedded, and sections were prepared and stained with PAS stain. The glomerular damage index (GDI) was calculated from 0 to 4 on the basis of the degree of glomerulosclerosis and mesangial matrix expansion, as previously described. ${ }^{35}$ In general, we counted 50 glomeruli in total in each kidney slice under a microscope, when each glomerulus was graded with level 0 to 4 damage, as follows: 0 represents no lesion; $1+$, sclerosis of $<25 \%$ of the glomerulus; $2+$, sclerosis of $25 \%$ to $50 \%$ of the glomerulus; $3+$, sclerosis of $>50 \%$ to $75 \%$ of the glomerulus; and $4+$, sclerosis of $>75 \%$ of the glomerulus. A whole kidney average sclerosis index was obtained by averaging scores from counted glomeruli. ${ }^{5}$ This observation was examined by two independent investigators (K.M.B. and M.X.) who were blinded to the treatment of the experimental groups. 


\section{Asm Activity in Mice Lacking Asm}

The activity of Asm was determined as previously described. ${ }^{36,37}$ Briefly, N-methyl-[ $\left.{ }^{14} \mathrm{C}\right]$-sphingomyelin was incubated with renal cortex tissue homogenates, and the metabolite of sphingomyelin, $\left[{ }^{14} \mathrm{C}\right]$-choline phosphate, was quantified. An aliquot of homogenates (20 $\mu \mathrm{g})$ was mixed with $0.02 \mu \mathrm{Ci}$ of $\mathrm{N}$-methyl-[ $\left[{ }^{14} \mathrm{C}\right]$-sphingomyelin in $100-\mu \mathrm{L}$ acidic reaction buffer containing $100 \mathrm{mmol} / \mathrm{L}$ sodium acetate and $0.1 \%$ Triton $\mathrm{X}-100, \mathrm{pH} 5.0$, and incubated at $37^{\circ} \mathrm{C}$ for 15 minutes. The reaction was terminated by adding $1.5 \mathrm{~mL}$ of chloroform-methanol (2:1) and $0.2 \mathrm{~mL}$ of double-distilled water. The samples were then vortex mixed and centrifuged at $1000 \times g$ for 5 minutes to separate into two phases. A portion of the upper aqueous phase containing $\left[{ }^{14} \mathrm{C}\right]$-choline phosphate was transferred to scintillation vials and counted in a Beckman liquid scintillation counter. The choline phosphate formation rate ( $\mathrm{nmol} / \mathrm{min} / \mathrm{mg}$ protein) was calculated to represent the enzyme activity.

\section{LC-Electrospray lonization Tandem MS for Quantitation of Ceramide}

The separation, identification, and quantitation of ceramide in plasma were performed by LC-mass spectrometry (MS). The HPLC instrument was equipped with a binary pump, a vacuum degasser, a thermostated column compartment, and an autosampler (Waters, Milford, $\mathrm{MA})$. The HPLC separations were performed at $70^{\circ} \mathrm{C}$ on an RP C18 Nucleosil AB column (5 $\mathrm{m}, 70 \times 2-\mathrm{mm}$ i.d.) from Macherey Nagel (Duren, Germany). The mobile phase was a gradient mixture formed as previously described. ${ }^{38}$ The plasma lipids were extracted according to previous studies. To avoid any loss of lipids, the whole procedure was performed in siliconized glassware. MS detection was performed using a Quattro II quadrupole MS instrument (Micromass, Altrincham, UK) operating under MassLynx 3.5 and configured with a Z-spray electrospray ionization source. Source conditions were previously described. ${ }^{4,38}$

\section{Cell Culture}

The conditionally immortalized mouse podocyte cell line, provided by Dr. P. E. Klotman (Division of Nephrology, Department of Medicine, Mount Sinai School of Medicine, New York, NY), was cultured on type I collagen-coated flasks or plates in RPMI 1640 medium supplemented with recombinant mouse interferon- $\gamma$ at $33^{\circ} \mathrm{C}$. After differentiation at $37^{\circ} \mathrm{C}$ for 10 to 14 days without interferon- $\gamma$, podocytes were used for the proposed experiments.

\section{Confocal Microscopic Detection of MR and Its Colocalization with Asm in Podocytes}

Podocytes were seeded on poly-L-lysine-coated chambers and treated with L-Hcys (40 $\mu \mathrm{mol} / \mathrm{L})$ or vehicle for 30 minutes. In an additional group of cells, amitriptyline (20 $\mu \mathrm{m}$; Sigma) was added to pretreat cells for 30 minutes before the addition of L-Hcys. The detection of membrane raft (MR) clusters and their colocalization were performed as previously described. Briefly, podocytes were washed with cold PBS, fixed for 15 minutes in $4 \%$ paraformaldehyde, and then blocked with $1 \%$ bovine serum albumin in PBS for 30 minutes. For detection of colocalization of MR and Asm, podocytes were first incubated with Alexa488labeled cholera toxin B at $1 \mu \mathrm{g} / \mathrm{mL}$ (Molecular Probes, Eugene, OR), as previously described. ${ }^{39}$ Then, the podocytes were incubated overnight with goat anti-Asm (1: 200; Santa Cruz Biotechnology, Santa Cruz, CA), followed by incubation with $5 \mu \mathrm{g} / \mathrm{mL}$ Texas Redconjugated anti-goat IgG for an additional 1 hour at room temperature. After mounting, the slides were observed using a confocal laser-scanning microscope (Fluoview FV1000; Olympus, Tokyo, Japan). ${ }^{39}$

\section{Urinary Total Protein and Albumin Excretion Measurements}

The 24-hour urine samples were collected using metabolic cages and subjected to total protein and albumin excretion measurements. ${ }^{31}$ The total protein content in the urine was detected by the Bradford method using a UV spectrophotometer. Urine albumin was detected using a commercially available albumin enzyme-linked immunosorbent assay kit (Bethyl Laboratories, Montgomery, TX). The urinary creatinine concentrations were measured by using a QuantiChrom creatinine assay kit (BioAssay System, Hayward, CA). ${ }^{40}$

\section{Delivery of Asm shRNA into the Kidneys by an Ultrasonograpic Microbubble Technique}

After a 1-week recovery period from uninephrectomy, Asm shRNA or a scrambled shRNA plasmid with a luciferase expression vector was cotransfected into the kidneys via intrarenal artery injection using the ultrasonographic microbubble system. A full description of the procedures for the ultrasonographic microbubble gene transfer technique can be found in our previous studies. ${ }^{2}$ To daily monitor the efficiency of gene expression through somatic plasmid transfection, mice were anesthetized with isoflurane and an aqueous solution of luciferin $(150 \mathrm{mg} / \mathrm{kg}$ ) was injected i.p. 5 minutes before imaging. The anesthetized mice were imaged using the IVIS200 in vivo molecular imaging system (Xenogen, Hopkinton, MA). Photons emitted from luciferase-expressing cells within the animal body and transmitted through tissue layers were quantified over a defined period, ranging up to 5 minutes, using the software program Living Image (Xenogen). The inhibitory efficiency of gene expression by Asm shRNA was further confirmed by detection of the Asm mRNA level in mouse renal cortex using real-time RT-PCR.

\section{ESR Analysis of $\mathrm{O}_{2} \cdot{ }^{\cdot-}$ Production}

For detection of Nox-dependent superoxide $\left(\mathrm{O}_{2}{ }^{-}\right)$ production, proteins from mouse renal cortex were extracted using sucrose buffer and resuspended with 
modified Krebs-HEPES buffer containing deferoximine (100 mmol/L; Sigma) and diethyldithiocarbamate (5 $\mathrm{mmol} / \mathrm{L}$; Sigma). The Nox-dependent $\mathrm{O}_{2}{ }^{\cdot-}$ production was examined by the addition of $1 \mathrm{mmol} / \mathrm{L} \mathrm{NADPH}$ as a substrate in $50 \mathrm{mg}$ of protein and incubated for $15 \mathrm{~min}$ utes at $37^{\circ} \mathrm{C}$ in the presence or absence of superoxide dismutase $(200 \mathrm{U} / \mathrm{mL})$ and then supplied with $1 \mathrm{mmol} / \mathrm{L}$ $\mathrm{O}_{2} \cdot{ }^{-}$-specific spin-trapping substance, 1-hydroxy-3-methoxycarbonyl-2,2,5,5-tetramethylpyrrolidine (Noxygen, Elzach, Germany). The mixture was loaded in glass capillaries and immediately analyzed for $\mathrm{O}_{2}{ }^{-}$production kinetically for 10 minutes in a Miniscope MS200 electromagnetic spin resonance (ESR) spectrometer (Magnettech Ltd, Berlin, Germany). The ESR settings were as follows: biofield, 3350; field sweep, 60 gauss; microwave frequency, $9.78 \mathrm{GHz}$; microwave power, $20 \mathrm{~mW}$; modulation amplitude, 3 gauss; 4096 points of resolution; receiver gain, 20 for tissue and 50 for cells. The results were expressed as the fold changes of control.

\section{Real-Time RT-PCR}

Total RNA from isolated mouse renal tissue was extracted using TRIzol reagent (Invitrogen, Carlsbad, CA), according to the protocol described by the manufacturer. RNA samples were quantified by measurement of optic absorbance at 260 and $280 \mathrm{~nm}$ in a spectrophotometer. The concentrations of RNA were calculated according to A260. Aliquots of total RNA (1 $\mu \mathrm{g})$ from each sample were reverse transcribed into cDNA, according to the instructions of the first-strand cDNA synthesis kit manufacturer (Bio-Rad, Hercules, CA). Equal amounts of the reverse transcriptional products were subjected to PCR amplification using SYBR Green as the fluorescence indicator on a Bio-Rad iCycler system (Bio-Rad). The primers used in this study were synthesized by Operon (Huntsville, $\mathrm{AL}$ ). The sequences were for the following: Asm, 5'-CACGTGGATGAGTTTGAGGT-3' (sense) and 5'-AGAGCTCCCAGAGTAGTTAC-3' (antisense); and $\beta$-actin, 5'-TCGCTGCGCTGGTCGTC-3' (sense) and 5'GGCCTCGTCACCCACATAGGA-3' (antisense). The mRNA levels of the target gene were normalized to the $\beta$-actin mRNA levels detected from the same samples. ${ }^{39}$

\section{Double-Immunofluorescent Staining}

Double-immunofluorescent staining was performed using frozen slides from mouse kidneys. After fixation, the slides were incubated with rabbit anti-podocin antibody at 1:100 (Sigma), which was followed by incubation with Alexa555-labeled goat anti-rabbit secondary antibody. Then, mouse anti-ceramide (1:50; Enzo Life Sciences, Plymouth Meeting, PA) or mouse anti-neutral sphingomyelinase (1:50; Santa Cruz Biotechnology) was used to incubate with the slides overnight at $4^{\circ} \mathrm{C}$. After washing, the slides were incubated with corresponding Alexa488labeled secondary antibodies. Finally, the slides were mounted and subjected to examinations using a confocal laser scanning microscope (Fluoview FV1000). All exposure settings were kept constant for each group of kidneys.

\section{Immunofluorescent Staining}

Immunofluorescent staining was performed using frozen slides of mouse kidneys. After fixation with acetone, the slides were incubated with anti-podocin (1:100; Sigma), anti-desmin (1:50; BD Biosciences, San Jose, CA), or anti-ceramide (1:50; Enzo Life Sciences) antibodies overnight at $4^{\circ} \mathrm{C}$. Then, these slides were washed and incubated with corresponding Texas Red-labeled secondary antibodies. Finally, the slides were washed, mounted, and subjected to fluorescent microscopy examination. The images were captured with a spot charge-coupled device camera and a pseudocolor was added to fluorescence showing in this slide (Diagnostic Instruments Inc., Sterlin Heights, MI). All exposure settings were kept constant for each group of kidneys.

\section{Statistical Analysis}

Data are provided as arithmetic mean \pm SEM; $n$ represents the number of independent experiments. All data were tested for significance using analysis of variance or paired and unpaired Student's $t$-test, as applicable. The GDI was analyzed using a nonparametric U-test. Only results with $P<0.05$ were considered statistically significant.

\section{Results}

\section{Asm Expression and Activity in the Kidneys of Mice Lacking the Asm Gene during hHcys}

As shown in Figure $1, A$ and $B$, Asm mRNA expression and its activity were significantly lower in $\mathrm{Asm}^{-1-}$ than in $\mathrm{Asm}^{+/+}$mice fed a normal diet. FF diet treatment significantly increased the Asm activity and mRNA expression in $\mathrm{Asm}^{+/+}$mice when compared with normal diet-fed mice. However, this FF diet-induced Asm activity and Asm mRNA expression were significantly attenuated in $\mathrm{Asm}^{-1-}$ mice (Figure 1, A and B). The total renal ceramide levels were similar in $\mathrm{Asm}^{+/+}$and $\mathrm{Asm}^{-1-}$ mice fed a normal diet. The FF diet significantly increased the total renal ceramide levels in $\mathrm{Asm}^{+/+}$mice but not in $\mathrm{Asm}^{-1-}$ mice (Figure 1C). Immunofluorescent analysis revealed that ceramide staining was more pronounced in glomeruli of FF diet-fed $\mathrm{Asm}^{+/+}$mice than in normal diet-fed mice. However, FF diet-induced ceramide production was less in $\mathrm{Asm}^{-/-}$mice (Figure 1D). In addition, confocal microscopic studies using podocin as a podocyte marker showed that hHcys-induced ceramide expression in glomeruli was mostly located in podocytes, as demonstrated by the colocalization of ceramide with podocin. This colocalization was substantially blocked in $\mathrm{Asm}^{-1-}$ mice (see Supplemental Figure S1 at http:// ajp.amjpathol.org). The FF diet did not alter the neutral sphingomyelinase expression in glomeruli of both $\mathrm{Asm}^{+/+}$and $\mathrm{Asm}^{-1-}$ mice (see Supplemental Figure S1 at $h$ ttp://ajp.amjpathol.org). The plasma Hcys concentration was similar in $\mathrm{Asm}^{+/+}$and $\mathrm{Asm}^{-/-}$mice. However, the FF diet significantly increased the plasma Hcys con- 
A

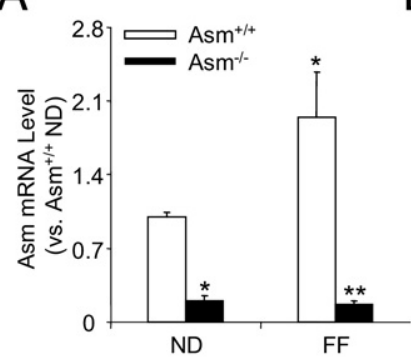

B

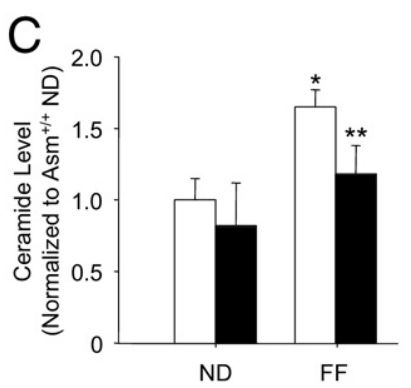

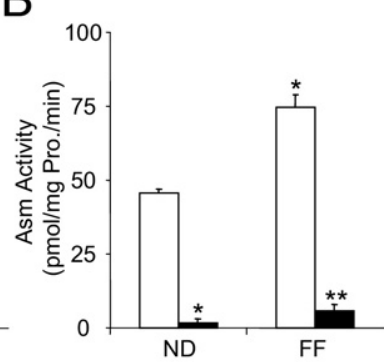

$\mathrm{D}$

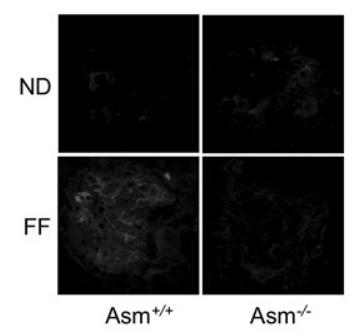

Figure 1. Effects of the normal diet (ND) and FF diet on renal tissue Asm mRNA, Asm activity, and total and glomerular ceramide production in $\mathrm{Asm}^{++}$and $\mathrm{Asm}^{-/-}$mice. Values are arithmetic mean $\pm \mathrm{SE}(n=4$ to 8 in each group) of Asm mRNA (A), Asm activity (B), total ceramide concentrations (C), and ceramide production (D) in $\mathrm{Asm}^{+/+}$and $\mathrm{Asm}^{-/-}$mice, with or without the FF diet. ${ }^{*} P<0.05$ versus values from $\mathrm{Asm}^{+/+}$mice fed the ND; ${ }^{* *} P<0.05$ versus values from mice receiving the FF diet.

centration in both mouse strains compared with that detected in mice receiving a normal diet $\left(\mathrm{Asm}^{+/+}\right.$mice, $37.6 \pm 5.9$ versus $4.1 \pm 0.41 \mu \mathrm{mol} / \mathrm{L}$ of control; and $\mathrm{Asm}^{-1-}$ mice, $38.4 \pm 8.6$ versus $4.0 \pm 0.8 \mu \mathrm{mol} / \mathrm{L}$ of control). Furthermore, we performed experiments to detect FF diet-induced Hcys levels in the renal tissue. The FF diet significantly increased the Hcys concentration in renal tissues of both $\mathrm{Asm}^{+/+}$(2.4-fold) and $\mathrm{Asm}^{-1-}$ (2.1fold) mice compared with the normal diet-fed $\mathrm{Asm}^{+/+}$ and $\mathrm{Asm}^{-1-}$ mice, respectively. These data confirm that the Asm gene is not involved in the regulation of plasma and renal tissue Hcys levels, and it does not alter the occurrence of hHcys in mice receiving the FF diet.

\section{Improvement of hHcys-Induced Glomerular Injury in Mice Lacking the Asm Gene}

As presented in Figure 2, A and B, urinary protein and albumin excretion was similar in $\mathrm{Asm}^{+/+}$and $\mathrm{Asm}^{-/-}$ mice fed a normal diet. However, an elevated plasma Hcys level, as the result of the FF diet, significantly increased the urinary total protein and albumin excretion in $\mathrm{Asm}^{+/+}$, but not in $\mathrm{Asm}^{-/-}$, mice. By PAS staining, we observed a typical pathological change in glomerular sclerotic damage in $\mathrm{Asm}^{+/+}$mice fed the FF diet, such as mesangial expansion and glomerular capillary collapse. This pathological feature was not observed in $\mathrm{Asm}^{-1-}$ mice. The average GDI was significantly higher in $\mathrm{Asm}^{+/+}$than in Asm ${ }^{-1-}$ mice when they were fed the FF diet (Figure 2, C and D).

An immunofluorescent histological analysis showed that desmin staining was more pronounced in glomeruli

of FF diet-fed Asm ${ }^{+/+}$mice than normal diet-fed mice, as shown by more remarkable red fluorescence detected with glomeruli, which indicates podocyte injury. The lack of Asm decreased the FF diet-induced increase in desmin expression within glomeruli of mice (Figure 3A). Desmin is an intermediate filament protein and may be an injured podocyte marker. The expression of desmin is often increased in various glomerular diseases in which podocyte damage is involved. ${ }^{5}$ Conversely, another podocyte marker, podocin, was markedly reduced in glomeruli of $\mathrm{Asm}^{+/+}$mice fed the FF diet compared with those fed the normal diet. However, this reduced podocin expression or production did occur in $\mathrm{Asm}^{-1-}$ mice, even receiving the FF diet (Figure $3 \mathrm{~B}$ ).

\section{Lack of Local Oxidative Stress in the Glomeruli of $h$ Hcys Mice Lacking the Asm Gene}

As illustrated in Figure 4A, the ESR spectrometric curve exhibited a significant increase in the amplitude of Noxdependent $\mathrm{O}_{2}{ }^{\cdot-}$ signals in the glomeruli of WT mice fed the FF diet compared with that observed in WT mice fed the normal diet. In Asm ${ }^{-1-}$ mice, hHcys induced by the FF diet failed to increase glomerular $\mathrm{O}_{2} \cdot{ }^{-}$production. These results were summarized in Figure 4B, showing that glomerular $\mathrm{O}_{2}{ }^{-}$- production was similar in $\mathrm{Asm}^{-1-}$ and $\mathrm{Asm}^{+/+}$mice fed the normal diet but increased by 2.5-fold in $\mathrm{Asm}^{+/+}$mice only when fed the FF diet. FF diet-induced glomerular $\mathrm{O}_{2} \cdot{ }^{-}$production was much less in $\mathrm{Asm}^{-1-}$ mice compared with $\mathrm{Asm}^{+/+}$mice.
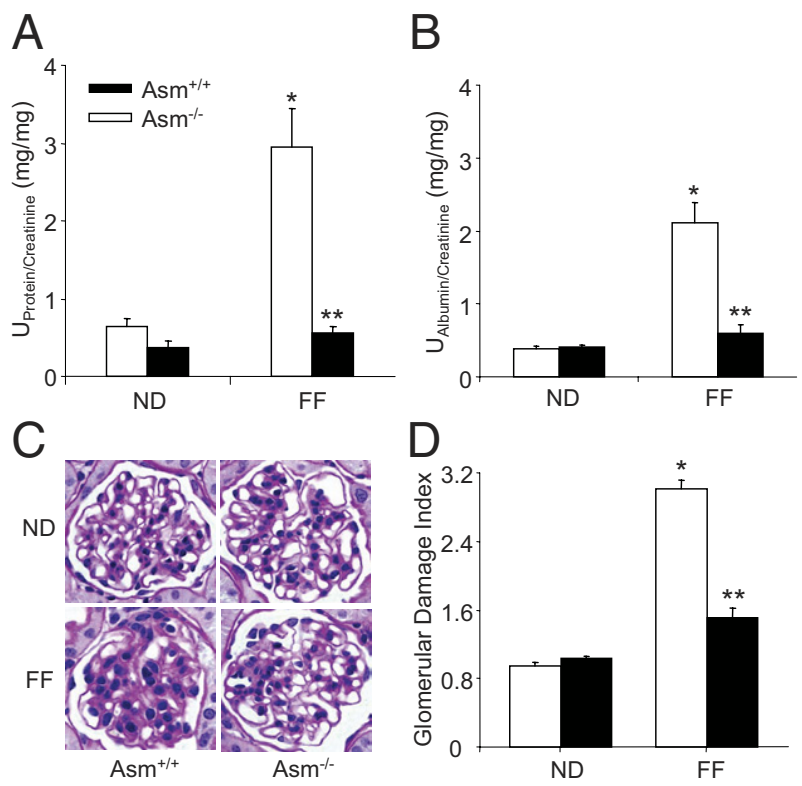

Figure 2. Effects of the normal diet (ND) and FF diet on glomerular injury in $\mathrm{Asm}^{+/+}$and $\mathrm{Asm}^{-1-}$ mice. A: Values are arithmetic mean \pm SEM $(n=4$ to 8 in each group) of urinary total protein excretion. B: Urinary albumin excretion in $\mathrm{Asm}^{+/+}$and $\mathrm{Asm}^{-/-}$mice, with or without the FF diet. C: Photomicrographs show typical glomerular structure (original magnification, $\times 400)$ in $\mathrm{Asm}^{+/+}$and Asm ${ }^{-/-}$mice, with or without the FF diet. D: Summarized data of GDI by semiquantitation of scores in four different groups of mice ( $n=6$ to 9 in each group). For each kidney section, 50 glomeruli were randomly chosen for the calculation of GDI. ${ }^{*} P<0.05$ versus the values from $\mathrm{Asm}^{+/+}$mice fed the ND; ${ }^{* *} P<0.05$ versus the values from mice fed the FF diet. 


\section{Decreased Asm Expression and Activity in the Kidney with Local Gene Silencing}

To further determine the implication of Asm in glomerular injury during hHcys, an shRNA strategy was used to locally silence this gene in the kidney and then observe the changes in glomerular function and pathological features during hHcys. As illustrated by images obtained by an in vivo molecular imaging system, luciferase gene expression cotransfected with Asm shRNA could be detected even on the third day after the kidney was transfected by ultrasonographic microbubble plasmid introduction. In the hemidissected kidney, all of the cortical regions ex-
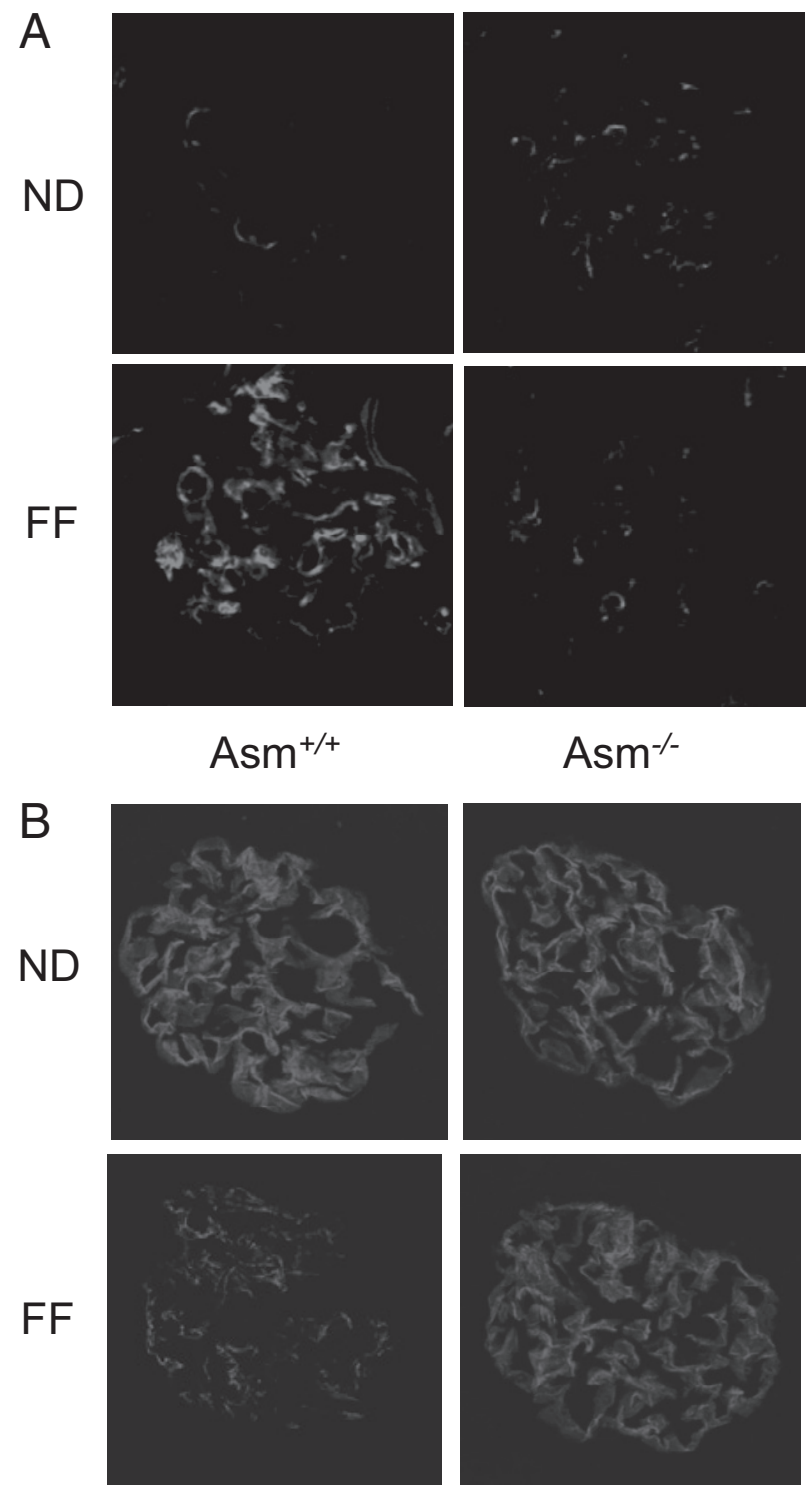

$\mathrm{Asm}^{-1-}$

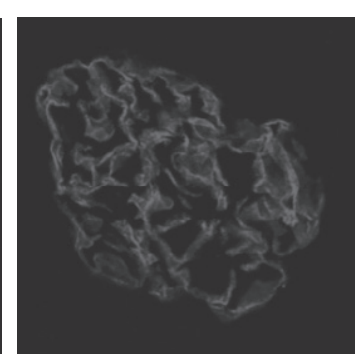

$\mathrm{Asm}^{+/+}$

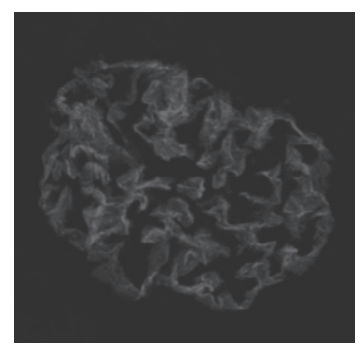

Asm ${ }^{-1-}$

Figure 3. Immunofluorescence staining of desmin and podocin in glomerul from $\mathrm{Asm}^{+/+}$and $\mathrm{Asm}^{-/-}$mice, with or without the FF diet. A: Typical images of desmin staining in glomeruli from Asm ${ }^{+/+}$and $\mathrm{Asm}^{-1-}$ mice, with or without the FF diet ( $n=6$ in each group). B: Typical images of podocin staining in glomeruli from Asm ${ }^{+/+}$and $\mathrm{Asm}^{-/-}$mice, with or without the FF diet ( $n=6$ in each group)
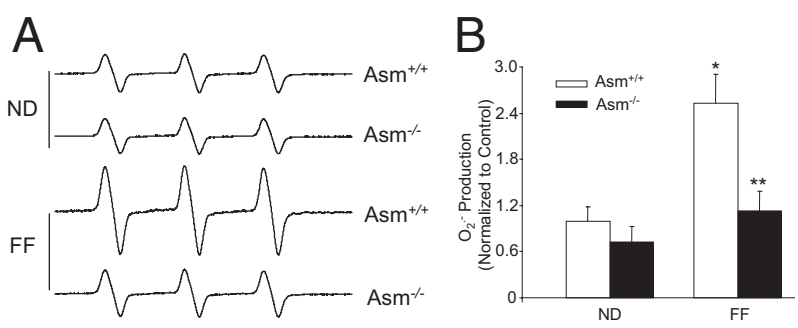

Figure 4. Effects of the normal diet (ND) and $\mathrm{FF}$ diet on glomerular $\mathrm{O}_{2}$. production in $\mathrm{Asm}^{+/+}$and $\mathrm{Asm}^{-/-}$mice. A: Representative ESR spectra traces for $\mathrm{O}_{2}{ }^{-}$production in $\mathrm{Asm}^{+/+}$and $\mathrm{Asm}^{-/-}$mice. B: Values are arithmetic mean $\pm \operatorname{SEM}\left(n=5\right.$ in each group) of $\mathrm{O}_{2} \cdot{ }^{-}$production in $\mathrm{Asm}^{+/+}$ and $\mathrm{Asm}^{-1-}$ mice fed with the ND or FF diet. ${ }^{*} P<0.05$ versus values from $\mathrm{Asm}^{+/+}$mice fed the ND; ${ }^{* * *} P<0.05$ versus values from mice fed the FF diet.

hibited efficient gene transfection, as shown in green and red fluorescence (Figure 5, A and $\mathrm{B}$ ). This is consistent with previous studies ${ }^{2,40}$ showing that ultrasonographic microbubble gene introduction is an efficient technique for delivery of the gene into the glomerular cells, vascular endothelial cells, and fibroblasts.
A in vivo

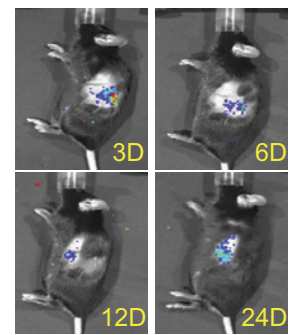

C

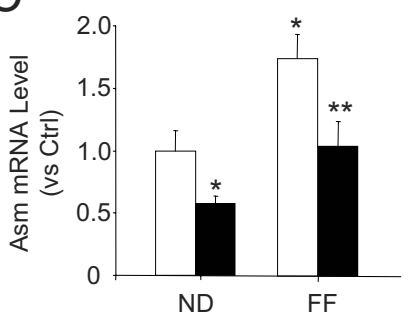

E

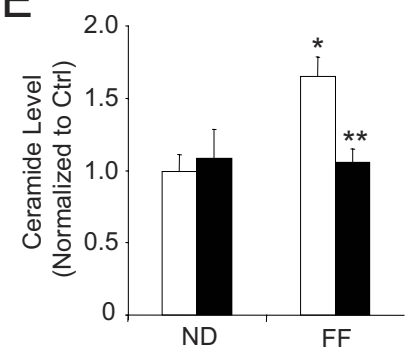

B
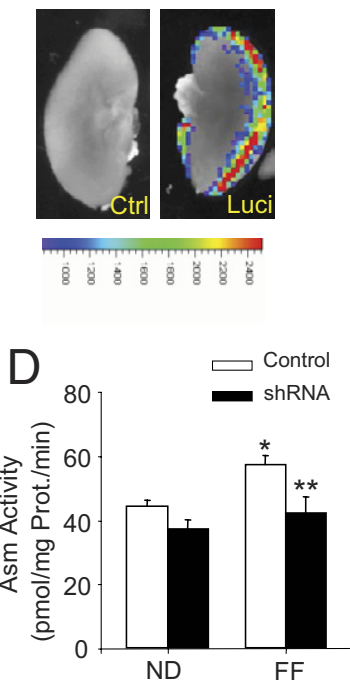

$\mathrm{F}$

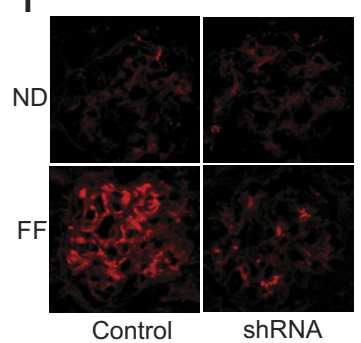

Figure 5. Renal Asm gene silencing efficiency in $\mathrm{C} 57 \mathrm{BL} / 6 \mathrm{~J}$ mice, with or without the FF diet. A: Daily imaging confirmation of gene transfection in the kidney by an in vivo molecular imaging system. D, days. B: Localization of transfected gene expression in the hemidissected kidney on day 12 after gene delivery. Ctrl, control; Luci, luciferase. Values are arithmetic mean \pm SEM ( $n=4$ to 8 in each group) of Asm mRNA expression (C), Asm activity (D), total ceramide concentrations $(\mathbf{E})$, and ceramide staining $(\mathbf{F})$ in ND- or FF diet-fed $\mathrm{C} 57 \mathrm{BL} / 6 \mathrm{~J}$ mice, with or without Asm shRNA transfection. ${ }^{*} P<0.05$ versus values from control mice fed the normal diet (ND); ${ }^{* * *} P<0.05$ versus values from mice fed the FF diet 
The efficiency of local Asm gene silencing was also examined by measurement of its expression and activity when animals were sacrificed after completion of functional studies. Real-time RT-PCR analysis demonstrated that Asm mRNA expression was significantly decreased in C57BL/6J WT mice transfected with Asm shRNA compared with control mice fed a normal diet. Compared with the normal diet, the FF diet significantly increased Asm mRNA expression in glomeruli from mice receiving scrambled shRNA, but it had no effect on Asm mRNA abundance in mice receiving Asm shRNA (Figure 5C). Correspondingly, the FF diet significantly increased the Asm activity in renal cortical tissue from mice receiving scrambled shRNA, but it was without effect in mice receiving Asm shRNA (Figure 5D). Moreover, the FF diet-induced increases in the total renal or glomerular ceramide level in mice receiving scrambled shRNA were not observed in mice receiving Asm shRNA (Figure 5, E and F).

\section{Blockade of $h$ Hcys-Induced Glomerular Injury by Asm Gene Silencing}

Similar to the results obtained from $\mathrm{Asm}^{-1-}$ mice, HPLC analysis demonstrated that local silencing of the ASM gene in the kidney had no significant effect on the plasma Hcys level. In mice receiving either scrambled or Asm shRNA, the FF diet significantly increased the plasma Hcys concentrations (data not shown). This confirms that Asm gene manipulation in the kidney does not alter the plasma Hcys concentration levels in mice.

We further determined whether Asm gene silencing locally in the kidney has any beneficial action on the FF diet-induced glomerular injury. As shown in Figure 6, A and $B$, urinary protein and albumin excretion was similar in both scrambled and Asm shRNA-transfected mice fed the normal diet. FF diet treatment significantly increased the urinary total protein and albumin excretion when compared with the normal diet-fed mice. However, the Asm shRNA transfection significantly attenuated FF diet-induced urinary total protein and albumin excretion. Morphological analysis showed a typical pathological change in glomerular sclerotic damage, such as an expanded glomerular mesangium with capillary collapse, hypercellularity, and fibrous deposition in mice fed the FF diet. The average GDI was significantly higher in FF diet-fed mice compared with normal diet-fed mice receiving scrambled shRNA. However, when Asm shRNA was transfected into the kidney, the FF diet-induced glomerular injuries were substantially attenuated (Figure 6, C and D).

By immunofluorescent microscopic analysis, desmin had more profound abundance in glomeruli of FF dietfed mice compared with that in normal diet-fed mice. Asm shRNA transfection abrogated the FF diet-induced increase in desmin expression (Figure 7A). In contrast, podocin was markedly reduced in glomeruli from FF dietfed mice compared with those from normal diet-fed mice. However, the Asm shRNA transfection in FF diet-fed mice completely attenuated the decrease in podocin staining (Figure 7B).
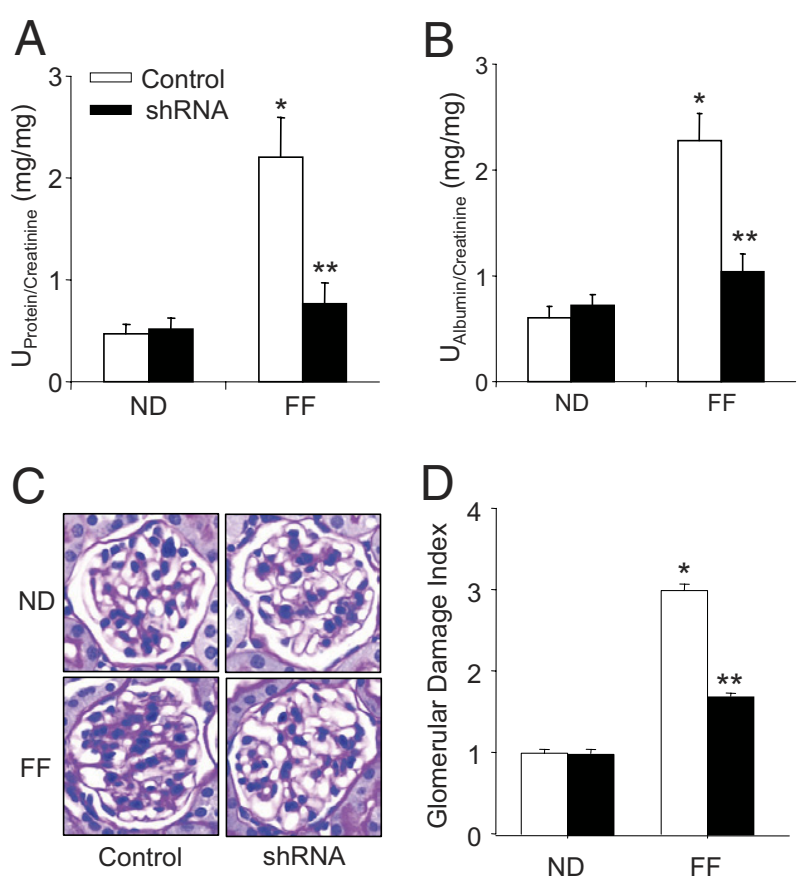

Figure 6. Effects of renal Asm silencing on glomerular injury in C57BL/6 mice, with or without the FF diet. A: Values are arithmetic mean \pm SEM ( $n=6$ to 8 in each group) of urinary total protein excretion. B: Urinary albumin excretion in normal diet (ND)- or FF diet-fed C57BL/6J mice, with or without Asm shRNA transfection. C: Photomicrographs show typical glomerular structure (original magnification, $\times 400$ ) during ND or FF diet treatment, with or without Asm shRNA transfection. D: Summarized data of GDI by semiquantitation of scores in four different groups of mice $(n=6$ in each group). For each kidney section, 50 glomeruli were randomly chosen for the calculation of GDI. " $P<0.05$ versus values from control mice fed the ND; ${ }^{* * *} P<0.05$ versus values from mice fed the FF diet.

\section{Suppression of hHcys-Induced Glomerular $\mathrm{O}_{2} \cdot-$ Production by Asm Gene Silencing}

As depicted in Figure 8, the glomerular $\mathrm{O}_{2}{ }^{--}$production was similar in both scrambled and Asm shRNA transfected mice when they were fed a normal diet. However, the FF diet significantly increased the glomerular $\mathrm{O}_{2}{ }^{-}$- production by 1.9-fold in scrambled shRNA transfected mice, but it had no significant effect on glomerular $\mathrm{O}_{2}{ }^{-1}$ production in mice with Asm shRNA transfection (Figure 8).

\section{Attenuation of L-Hcys-Induced MR Clustering in Podocytes by Asm Inhibition}

In addition, we determined the colocalization of MR and Asm in the plasma membrane on Hcys stimulation. In this regard, the podocytes were treated with Texas Red-antiAsm and fluorescein isothiocyanate-cholera toxin $\mathrm{B}$, and the distribution of anti-Asm within MR clusters was visualized in the podocyte membrane by confocal microscopy. Under the control condition, Asm was evenly spread throughout the whole cell. On Hcys stimulation, Asm translocated to the membrane, as shown by red fluorescence spots or patches. Yellow patches or dots in the overlaid images were considered a colocalization of Asm in MR clusters. Pretreatment of podocytes with the Asm inhibitor, amitriptyline, led to the disruption of MR and abrogated 


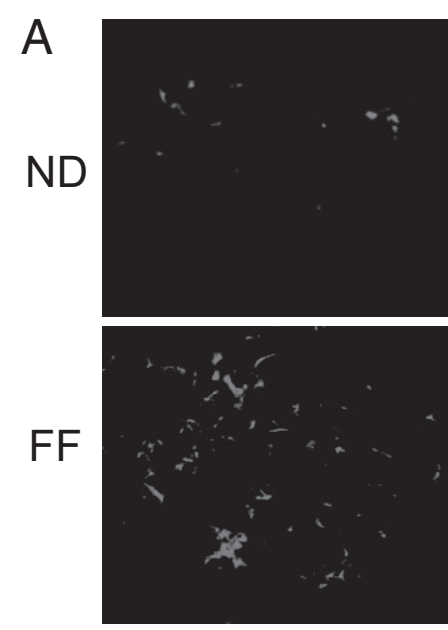

Control
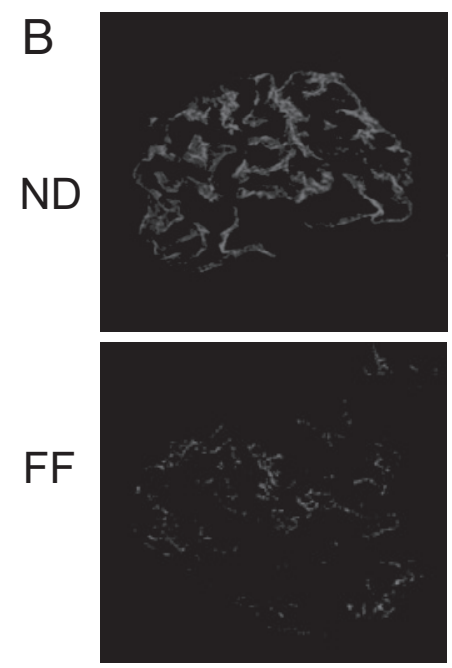

Control
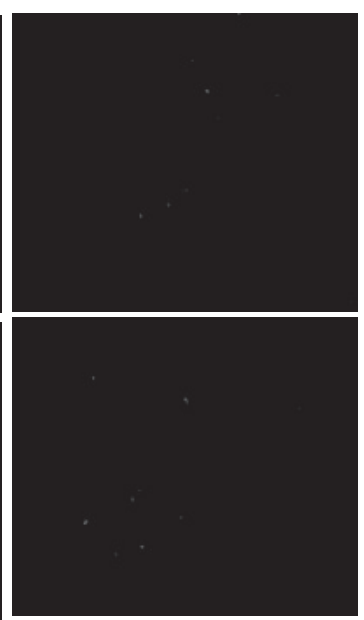

shRNA
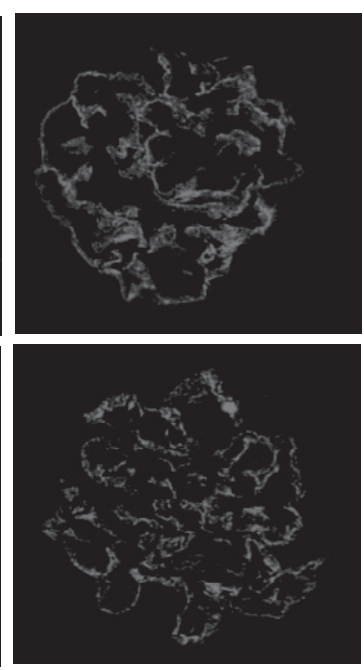

shRNA
Figure 7. Effects of renal Asm gene silencing on the expression of desmin and podocin in glomeruli in $\mathrm{C} 57 \mathrm{BL} / 6 \mathrm{~J}$ mice, with or without the FF diet. $\mathbf{A}$ : Typical images of desmin staining in glomeruli from C57BL/6J mice fed the normal diet (ND) or FF diet, with or without Asm shRNA transfection ( $n=6$ in each group). B: Typical images of podocin staining in glomeruli from C57BL/6J mice fed the ND or FF diet, with or without Asm shRNA transfection ( $n=6$ in each group).

patching and clustering of both fluorescein isothiocyanatecholera toxin B and Asm after Hcys stimulation (see Supplemental Figure S2 at http://ajp.amjpathol.org).

\section{Discussion}

The goal of the present study is to determine whether Asm, a ceramide-producing enzyme, is implicated in the development of hHcys-induced glomerular oxidative stress and injury. We found that FF diet treatment enhanced the Asm activity, Asm mRNA expression, and ceramide production, which was attributed to Nox-dependent $\mathrm{O}_{2} \cdot-$ production and local oxidative stress in glomeruli and ultimately led to glomerulosclerosis. Our results demonstrate, for the first time to our knowledge, that hHcys-induced glomerular oxidative stress and injury were attenuated by Asm gene deficiency in mice.

To examine the role of Asm in mediating hHcys-induced glomerular injury or sclerosis, we first induced hHcys in Asm ${ }^{-1-}$ mice and their WT littermates by feeding them an FF diet for 8 weeks. FF diet treatment significantly increased plasma and renal tissue Hcys levels in both $\mathrm{Asm}^{+/+}$and $\mathrm{Asm}^{-1-}$ mice, indicating successful establishment of the hHcys and also suggesting that Asm itself is not involved in the global metabolism of Hcys. The increased plasma and renal tissue Hcys concentration resulted in remarkable glomerular damage or sclerosis in $\mathrm{Asm}^{+/+}$, but not in $\mathrm{Asm}^{-/-}$, mice, suggesting that the lack of the Asm gene leads to blockade of pathogenic pathways mediating hHcys-induced glomerular injury.

Earlier studies reported that ceramide has been implicated in the regulation of kidney function. Ceramide as a potent regulator of cell proliferation, activation, and apoptosis plays an important role in lipoprotein aggregation and may promote foam cell formation in atherosclerosis. ${ }^{41}$ This lipid mediator has mediated the detrimental or pathogenic actions of many different injury factors in different cells and tissues. ${ }^{12,41,42}$ In this regard, there are reports ${ }^{43}$ that serine palmitoyl-CoA transferase inhibition by myriocin leads to a reduction of plasma sphingomyelin, ceramide production, and atherosclerosis in apolipoprotein E-knockout mice. We also demonstrated by pharmacological interventions that ceramide contributes to the development of chronic glomerular injury associated with hHcys and obesity. 4,31 The present study further demonstrated that Asm gene deficiency attenuated hHcys-increased ceramide production in the kidney and thereby blocked glomerular injury associated with hHcys. Profiling analysis of renal ceramide demonstrated that, although $\mathrm{C}_{24}$ ceramide constituted the major ceramide component that practically determines total ceramide levels, the most dramatic changes in response to FF diet-induced hHcys were observed from less-dominant ceramide isoforms in mice. Correspondingly, local glomerular ceramide was more abundant in $\mathrm{Asm}^{+/+}$than in $\mathrm{Asm}^{-1-}$ mice when they both were fed the FF diet to induce hHcys. Furthermore, Asm activity and Asm mRNA expression in renal tissues were significantly increased in

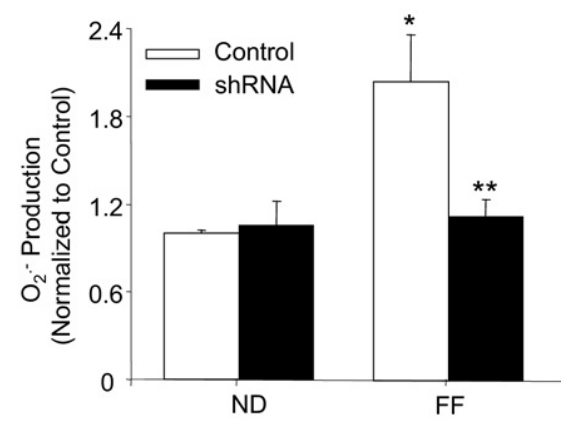

Figure 8. Effect of renal Asm gene silencing on glomerular $\mathrm{O}_{2}{ }^{-}$production in $\mathrm{C} 57 \mathrm{BL} / 6 \mathrm{~J}$ mice, with or without the FF diet. Values are arithmetic mean \pm SEM ( $n=5$ to 6 each group) of $\mathrm{O}_{2}{ }^{-}$production in $\mathrm{C} 57 \mathrm{BL} / 6 \mathrm{~J}$ mice fed the normal diet (ND) or FF diet with or without Asm shRNA transfection. ${ }^{*} P<$ 0.05 versus values from control mice fed the ND; ${ }^{* * *} P<0.05$ versus values from mice fed the FF diet. 
FF diet-fed Asm ${ }^{+/+}$mice but not in $\mathrm{Asm}^{-/-}$mice. In addition, our studies demonstrated that Hcys stimulation enhanced the colocalization of MR and Asm in the plasma membrane and confirmed the translocation of Asm into the cell membrane. These results together suggest that FF diet-induced hHcys increases the renal and glomerular ceramide levels, mainly because of activation of Asm and an increase in its expression.

Furthermore, we demonstrated that decreased ceramide production via Asm has a protective role in the glomerular injury associated with hHcys. In accordance with lowered ceramide production in $\mathrm{Asm}^{-1-}$ mice fed the FF diet, urinary albumin, protein excretion, and glomerular podocyte injury and sclerosis were also significantly decreased compared with $\mathrm{Asm}^{+/+}$mice fed the FF diet, suggesting that ceramide-associated renal injury during hHcys is alleviated in these Asm gene knockout mice. Therefore, this sphingomyelinase could be a target of a therapeutic strategy for hHcys-induced glomerular injury or sclerosis.

To further explore the mechanism of glomerular injury during hHcys, we observed changes in podocyte function in $\mathrm{Asm}^{+/+}$and $\mathrm{Asm}^{-/-}$mice. It has been well documented that podocyte loss and dysfunction occur with the onset and magnitude of glomerulosclerosis. Because podocytes serve as the final barrier against urinary protein loss in the normal glomeruli, any change in podocyte structure and function may be intimately associated with proteinuria and consequent glomerular sclerosis. ${ }^{44}$ The present study showed that podocin protein was markedly decreased in FF diet-fed $\mathrm{Asm}^{+/+}$mice but not in mice lacking Asm. In addition, we found that desmin, an intermediate filament protein and a specific and sensitive podocyte injury marker, was increased in the glomeruli when $\mathrm{Asm}^{+/+}$mice received the FF diet. This increased desmin expression in the glomeruli was attenuated in FF diet-fed $\mathrm{Asm}^{-1-}$ mice. These results further support the view that hHcys-induced glomerular injury is associated with increased ceramide production via Asm.

Reportedly, oxidative stress has been implicated in the development of glomerular injury and end-stage renal disease. In our previous studies, ${ }^{4}$ Nox-derived $\mathrm{O}_{2} \cdot$ - production was an important mechanism mediating hHcys-induced glomerular injury or damage. NA$\mathrm{DPH}$-dependent $\mathrm{O}_{2}{ }^{-}$- production is an early event for homocysteine-induced glomerular cell damage and glomerular sclerosis. ${ }^{4,36}$ It is possible that hHcys-induced Nox activation is mediated by enhanced Asm activity. The present study hypothesized that Asm may be associated with local oxidative stress in the glomeruli of mice with hHcys. Indeed, electron-spin resonance analysis showed that FF diet treatment significantly increased Nox-dependent $\mathrm{O}_{2}{ }^{--}$production in $\mathrm{Asm}^{+/+}$, but not in $\mathrm{Asm}^{-1-}$, mice. These results confirm the imperative role of Asm in mediating $\mathrm{O}_{2}{ }^{-}$- production through the activation of Nox in the glomeruli during hHcys.

To further address the role of the Asm gene in mediating hHcys-induced podocyte and glomerular injury, a local gene-silencing strategy was used in the present study, in which ultrasonographic microbubble-mediated plasmid delivery was used to introduce Asm shRNA into the kidney. This method was highly efficient in delivering plasmids into renal cells in vivo, which led to gene transfection and expression in most renal cells (90\%), as confirmed by earlier reports. ${ }^{2,45-48}$ By using an in vivo molecular imaging system to daily monitor the efficiency of Asm gene transfection in the kidney in living animals, we showed that the transgene or shRNA expression vector (with the luciferase gene as an indicator) could be detected even 3 days after gene transfection and lasted for 4 weeks by observation. This in vivo transgene monitoring importantly guided our functional studies to define the role of the Asm gene in mediating glomerular damage associated with hHcys. After completion of functional protocols, Asm mRNA expression, Asm activity, and renal and glomerular ceramide production were analyzed to confirm the efficient silencing of the Asm gene in shRNA-transfected kidneys. In such local Asm genesilenced kidney, we found that the glomerular ceramide level was significantly decreased and that $\mathrm{O}_{2}{ }^{-}{ }^{-}$production was markedly reduced. Silencing the Asm gene in the kidney ameliorates proteinuria/albuminuria and podocyte injury, whereby glomerular sclerotic changes were substantially suppressed. These results from mice with local renal Asm gene silencing further support the previous conclusion, drawn from studies using Asm-knockout mice that Asm is importantly implicated in the development of podocytes and glomerular injury. The reduction of ceramide production or the inhibition of Nox is preventive to hHcys-induced glomerular injury. Further studies with a careful experimental design for therapeutic action via this signaling system are needed to confirm its therapeutic potential. In conclusion, the present study demonstrated that Asm gene deficiency attenuates the $\mathrm{hH}$ cys-induced glomerular oxidative stress and injury. The amelioration of glomerular injury by Asm deficiency during hHcys implicates the pivotal role of Asm in hHcysinduced glomerulosclerosis.

\section{References}

1. Dennis VW, Robinson K: Homocysteinemia and vascular disease in end-stage renal disease. Kidney Int Suppl 1996, 57:S11-S17

2. Yi F, Xia M, Li N, Zhang C, Tang L, Li PL: Contribution of guanine nucleotide exchange factor Vav2 to hyperhomocysteinemic glomerulosclerosis in rats. Hypertension 2009, 53:90-96

3. Ingram AJ, Krepinsky JC, James L, Austin RC, Tang D, Salapatek AM, Thai K, Scholey JW: Activation of mesangial cell MAPK in response to homocysteine. Kidney Int 2004, 66:733-745

4. Yi F, Zhang AY, Li N, Muh RW, Fillet M, Renert AF, Li PL: Inhibition of ceramide-redox signaling pathway blocks glomerular injury in hyperhomocysteinemic rats. Kidney Int 2006, 70:88-96

5. Zhang C, Hu JJ, Xia M, Boini KM, Brimson CA, Laperle LA, Li PL: Protection of podocytes from hyperhomocysteinemia-induced injury by deletion of the gp91phox gene. Free Radic Biol Med 2010, 48: 1109-1117

6. Tyagi N, Moshal KS, Sen U, Vacek TP, Kumar M, Hughes WM Jr, Kundu S, Tyagi SC: H2S protects against methionine-induced oxidative stress in brain endothelial cells. Antioxid Redox Signal 2009, 11:25-33

7. Hwang SY, Siow YL, Au-Yeung KK, House J, OK: Folic acid supplementation inhibits NADPH oxidase-mediated superoxide anion production in the kidney. Am J Physiol Renal Physiol 2011, 300:F189-F198

8. Yi F, Li PL: Mechanisms of homocysteine-induced glomerular injury and sclerosis. Am J Nephrol 2008, 28:254-264 
9. Li PL, Yi F, Li N: Hyperhomocysteinemia: association with renal transsulfuration and redox signaling in rats. Clin Chem Lab Med 2007, 45:1688-1693

10. Papatheodorou L, Weiss $N$ : Vascular oxidant stress and inflammation in hyperhomocysteinemia. Antioxid Redox Signal 2007, 9:1941-1958

11. Kaushal GP, Singh AB, Shah SV: Identification of gene family of caspases in rat kidney and altered expression in ischemia-reperfusion injury. Am J Physiol 1998, 274:F587-F595

12. Ueda N, Kaushal GP, Shah SV: Apoptotic mechanisms in acute renal failure. Am J Med 2000, 108:403-415

13. Yin T, Sandhu G, Wolfgang CD, Burrier A, Webb RL, Rigel DF, Hai T, Whelan J: Tissue-specific pattern of stress kinase activation in ischemic/reperfused heart and kidney. J Biol Chem 1997, 272:1994319950

14. Zhang CE, Wei W, Liu YH, Peng JH, Tian Q, Liu GP, Zhang Y, Wang $\mathrm{JZ}$ : Hyperhomocysteinemia increases beta-amyloid by enhancing expression of gamma-secretase and phosphorylation of amyloid precursor protein in rat brain. Am J Pathol 2009, 174:1481-1491

15. Schmitz-Peiffer C: Targeting ceramide synthesis to reverse insulin resistance. Diabetes 2010, 59:2351-2353

16. Ussher JR, Koves TR, Cadete VJ, Zhang L, Jaswal JS, Swyrd SJ, Lopaschuk DG, Proctor SD, Keung W, Muoio DM, Lopaschuk GD: Inhibition of de novo ceramide synthesis reverses diet-induced insulin resistance and enhances whole-body oxygen consumption. Diabetes 2010, 59:2453-2464

17. Mielke MM, Haughey NJ, Ratnam Bandaru VV, Schech S, Carrick R, Carlson MC, Mori S, Miller MI, Ceritoglu C, Brown T, Albert M, Lyketsos CG: Plasma ceramides are altered in mild cognitive impairment and predict cognitive decline and hippocampal volume loss. Alzheimers Dement 2010, 6:378-385

18. Holland WL, Brozinick JT, Wang LP, Hawkins ED, Sargent KM, Liu Y Narra K, Hoehn KL, Knotts TA, Siesky A, Nelson DH, Karathanasis SK, Fontenot GK, Birnbaum MJ, Summers SA: Inhibition of ceramide synthesis ameliorates glucocorticoid-, saturated-fat-, and obesityinduced insulin resistance. Cell Metab 2007, 5:167-179

19. Summers SA: Ceramides in insulin resistance and lipotoxicity. Prog Lipid Res 2006, 45:42-72

20. Futerman $\mathrm{AH}$, Hannun YA: The complex life of simple sphingolipids EMBO Rep 2004, 5:777-782

21. Garcia-Barros M, Paris F, Cordon-Cardo C, Lyden D, Rafii S, Haimovitz-Friedman A, Fuks Z, Kolesnick R: Tumor response to radiotherapy regulated by endothelial cell apoptosis. Science 2003, 300: 1155-1159

22. Smith EL, Schuchman EH: The unexpected role of acid sphingomyelinase in cell death and the pathophysiology of common diseases. FASEB J 2008, 22:3419-3431

23. Grassme H, Cremesti A, Kolesnick R, Gulbins E: Ceramide-mediated clustering is required for CD95-DISC formation. Oncogene 2003 22:5457-5470

24. Yu ZF, Nikolova-Karakashian M, Zhou D, Cheng G, Schuchman EH, Mattson MP: Pivotal role for acidic sphingomyelinase in cerebra ischemia-induced ceramide and cytokine production, and neurona apoptosis. J Mol Neurosci 2000, 15:85-97

25. Paris F, Fuks Z, Kang A, Capodieci P, Juan G, Ehleiter D, HaimovitzFriedman A, Cordon-Cardo C, Kolesnick R: Endothelial apoptosis as the primary lesion initiating intestinal radiation damage in mice. Science 2001, 293:293-297

26. Dimanche-Boitrel MT, Meurette O, Rebillard A, Lacour S: Role of early plasma membrane events in chemotherapy-induced cell death. Drug Resist Updat 2005, 8:5-14

27. Garcia-Ruiz C, Colell A, Mari M, Morales A, Calvo M, Enrich C Fernandez-Checa JC: Defective TNF-alpha-mediated hepatocellular apoptosis and liver damage in acidic sphingomyelinase knockout mice. J Clin Invest 2003, 111:197-208

28. Dhami R, He X, Schuchman EH: Acid sphingomyelinase deficiency attenuates bleomycin-induced lung inflammation and fibrosis in mice. Cell Physiol Biochem 2010, 26:749-760

29. Becker KA, Riethmuller J, Luth A, Doring G, Kleuser B, Gulbins E: Acid sphingomyelinase inhibitors normalize pulmonary ceramide and inflammation in cystic fibrosis. Am J Respir Cell Mol Biol 2010 42:716-724

30. Teichgraber V, Ulrich M, Endlich N, Riethmuller J, Wilker B, OliveiraMunding CC, van Heeckeren AM, Barr ML, von Kurthy G, Schmid KW, Weller M, Tummler B, Lang F, Grassme H, Doring G, Gulbins E: Ceramide accumulation mediates inflammation, cell death and infection susceptibility in cystic fibrosis. Nat Med 2008, 14:382-391

31. Boini KM, Zhang C, Xia M, Poklis JL, Li PL: Role of sphingolipid mediator ceramide in obesity and renal injury in mice fed a high-fat diet. J Pharmacol Exp Ther 2010, 334:839-846

32. Moles A, Tarrats N, Morales A, Dominguez M, Bataller R, Caballeria J, Garcia-Ruiz C, Fernandez-Checa JC, Mari M: Acidic sphingomyelinase controls hepatic stellate cell activation and in vivo liver fibrogenesis. Am J Pathol 2010, 177:1214-1224

33. Achar E, Maciel TT, Collares CF, Teixeira VP, Schor N: Amitriptyline attenuates interstitial inflammation and ameliorates the progression of renal fibrosis. Kidney Int 2009, 75:596-604

34. Chen YF, Li PL, Zou AP: Effect of hyperhomocysteinemia on plasma or tissue adenosine levels and renal function. Circulation 2002, 106 1275-1281

35. Raij L, Azar S, Keane W: Mesangial immune injury, hypertension, and progressive glomerular damage in Dahl rats. Kidney Int 1984, 26 : 137-143

36. Yi F, Zhang AY, Janscha JL, Li PL, Zou AP: Homocysteine activates $\mathrm{NADH} / \mathrm{NADPH}$ oxidase through ceramide-stimulated Rac GTPase activity in rat mesangial cells. Kidney Int 2004, 66:1977-1987

37. Boini KM, Zhang C, Xia M, Han WQ, Brimson C, Poklis JL, Li PL: Visfatin-induced lipid raft redox signaling platforms and dysfunction in glomerular endothelial cells. Biochim Biophys Acta 2010, 1801: 1294-1304

38. Fillet M, Van Heugen JC, Servais AC, De Graeve J, Crommen J: Separation, identification and quantitation of ceramides in human cancer cells by liquid chromatography-electrospray ionization tandem mass spectrometry. J Chromatogr A 2002, 949:225-233

39. Zhang C, Hu JJ, Xia M, Boini KM, Brimson C, Li PL: Redox signaling via lipid raft clustering in homocysteine-induced injury of podocytes. Biochim Biophys Acta 2010, 1803:482-491

40. van der Wouden EA, Sandovici M, Henning $\mathrm{RH}$, de Zeeuw D, Deelman LE: Approaches and methods in gene therapy for kidney disease. J Pharmacol Toxicol Methods 2004, 50:13-24

41. Williams KJ, Tabas I: The response-to-retention hypothesis of early atherogenesis. Arterioscler Thromb Vasc Biol 1995, 15:551-561

42. Coroneos E, Martinez M, McKenna S, Kester M: Differential regulation of sphingomyelinase and ceramidase activities by growth factors and cytokines: implications for cellular proliferation and differentiation. J Biol Chem 1995, 270:23305-23309

43. Hojjati MR, Li Z, Zhou H, Tang S, Huan C, Ooi E, Lu S, Jiang XC: Effect of myriocin on plasma sphingolipid metabolism and atherosclerosis in apoE-deficient mice. J Biol Chem 2005, 280:10284-10289

44. Li N, Chen L, Yi F, Xia M, Li PL: Salt-sensitive hypertension induced by decoy of transcription factor hypoxia-inducible factor-1alpha in the renal medulla. Circ Res 2008, 102:1101-1108

45. Hou CC, Wang W, Huang XR, Fu P, Chen TH, Sheikh-Hamad D, Lan HY: Ultrasound-microbubble-mediated gene transfer of inducible Smad7 blocks transforming growth factor-beta signaling and fibrosis in rat remnant kidney. Am J Pathol 2005, 166:761-771

46. Koike H, Tomita N, Azuma H, Taniyama Y, Yamasaki K, Kunugiza Y, Tachibana K, Ogihara T, Morishita R: An efficient gene transfer method mediated by ultrasound and microbubbles into the kidney. J Gene Med 2005, 7:108-116

47. Lan HY, Mu W, Tomita N, Huang XR, Li JH, Zhu HJ, Morishita R, Johnson RJ: Inhibition of renal fibrosis by gene transfer of inducible Smad7 using ultrasound-microbubble system in rat UUO model. J Am Soc Nephrol 2003, 14:1535-1548

48. Sheyn D, Kimelman-Bleich N, Pelled G, Zilberman Y, Gazit D, Gazit Z: Ultrasound-based nonviral gene delivery induces bone formation in vivo. Gene Ther 2008, 15:257-266 